KYUNGPOOK Math. J. 53(2013), 459-465

http://dx.doi.org/10.5666/KMJ.2013.53.3.459

\title{
Differential Subordination Properties of Sokól-Stankiewicz Starlike Functions
}

\author{
RASHIDAH OMAR* \\ Institute of Mathematical Sciences, Faculty of Science, University of Malaya \\ Malaysia \\ Faculty of Computer and Mathematical Sciences, MARA University of Technology \\ Malaysia \\ e-mail : Ashidah@hotmail.com \\ Suzeini AbDul Halim \\ Institute of Mathematical Sciences, Faculty of Science, University of Malaya \\ Malaysia \\ e-mail : suzeini@um.edu.my
}

ABStract. Let $p(z)$ be an analytic function defined on the open unit disk $\mathbf{D}$ and $p(0)=1$. Condition $\beta$ in terms of complex numbers $D$ and real $E$ with $-1<E<1$ and $|D| \leq 1$ is determined such that $1+\beta z p^{\prime}(z) \prec \frac{1+D z}{1+E z}$ implies $p(z) \prec \sqrt{1+z}$. Furthermore, the expression $1+\frac{\beta z p^{\prime}(z)}{p(z)}$ and $1+\frac{\beta z p^{\prime}(z)}{p^{2}(z)}$ are considered in obtaining similar results.

\section{Introduction}

Let $A$ denote the class of all analytic functions $f$ in the open unit disk $\mathbf{D}:=\{z \in \mathbf{C}:|z|<1\}$ and normalised by $f(0)=0, f^{\prime}(0)=1$. An analytic function $f$ is subordinate to an analytic function $g$, written $f(z) \prec g(z)(z \in \mathbf{D})$, if there exists an analytic function $w$ in $\mathbf{D}$ such that $w(0)=0$ and $|w(z)|<1$ for $|z|<1$ and $f(z)=g(w(z))$. In particular, if $g$ is univalent in $\mathbf{D}$, then $f(z) \prec g(z)$ is equivalent to $f(0)=g(0)$ and $f(\mathbf{D}) \subset g(\mathbf{D})$.

Sokół and Stankiewicz [6] introduced the class $S L^{\star}$ consisting of normalised analytic functions $f$ in $\mathbf{D}$ satisfying the condition $\left|\left[\frac{z f^{\prime}(z)}{f(z)}\right]^{2}-1\right|<1, z \in \mathbf{D}$. Geometrically, a function $f \in S L^{\star}$ if $\frac{z f^{\prime}(z)}{f(z)}$ is in the interior of the right half of the lemniscate of Bernoulli $\left(x^{2}+y^{2}\right)^{2}-2\left(x^{2}-y^{2}\right)=0$. A function in the class $S L^{\star}$ is

* Corresponding Author.

Received August 2, 2011; accepted August 22, 2012.

2010 Mathematics Subject Classification: 30C45, 30C50 .

Key words and phrases: Analytic functions, differential subordination, Sokól-Stankiewicz starlike functions. 
called Sokół-Stankiewicz starlike function. Alternatively, we can also write

$$
f \in S L^{\star} \Leftrightarrow \frac{z f^{\prime}(z)}{f(z)} \prec \sqrt{1+z} .
$$

Properties of functions in $S L^{\star}$ have intensively been studied by authors in [4], [7], [8], [9] and [10].

Next, we denote $S^{\star}[A, B]$ as the class of Janowski starlike functions introduced by Janowski [1] and it consists of functions $f \in A$ satisfying

$$
\frac{z f^{\prime}(z)}{f(z)} \prec \frac{1+A z}{1+B z} \quad(-1 \leq B<A \leq 1) .
$$

For analytic function $p(z)$ in $\mathbf{D}$ with $p(0)=1$, Nunokawa et. al. [3] investigated and established the relation $1+z p^{\prime}(z) \prec 1+z$ implies $p(z) \prec 1+z$. Ali et. al. [5] extended this result and obtained conditions for which $1+z p^{\prime}(z) \prec \frac{1+D z}{1+E z}$ implies $p(z) \prec \frac{1+A z}{1+B z}$. Recently, in [4], condition for which $1+z p^{\prime}(z) \prec \sqrt{1+z}$ implies $p(z) \prec \sqrt{1+z}$ were determined. Motivated by these studies, this paper considers ascertaining condition so that $1+z p^{\prime}(z) \prec \frac{1+D z}{1+E z}$ implies $p(z) \prec \sqrt{1+z}$. Other results involving the expression $1+\frac{\beta z p^{\prime}(z)}{p(z)}$ and $1+\frac{\beta z p^{\prime}(z)}{p^{2}(z)}$ were also looked at.

\section{Main Results}

In proving our results, the following lemma proved by Miller and Mocanu is used.

Lemma 2.1([2], p. 135. Let $q$ be univalent in $\boldsymbol{D}$ and let $\varphi$ be analytic in a domain containing $q(\boldsymbol{D})$. Let $z q^{\prime}(z) \varphi[q(z)]$ be starlike. If $p$ is analytic in $D, p(0)=q(0)$ and satisfies $z p^{\prime}(z) \varphi[p(z)] \prec z q^{\prime}(z) \varphi[q(z)]$ then $p \prec q$ and $q$ is the best dominant.

Our first result is as follows:

Theorem 2.1. Let $p$ be an analytic function on $\boldsymbol{D}$ and $p(0)=1$.

Let $\beta \geq \beta_{0}, \beta_{0}=\frac{2 \sqrt{2}|D-E|}{(1-|E|)}$ where $-1<E<1$ and $|D| \leq 1$.

If

$$
1+\beta z p^{\prime}(z) \prec \frac{1+D z}{1+E z}
$$

then

$$
p(z) \prec \sqrt{1+z} .
$$

Proof. Let $q(z)=\sqrt{1+z}$ with $q(0)=1, q: \mathbf{D} \rightarrow C$. Since $q(\mathbf{D})$ is a convex set thus $q$ is a convex function which implies $z q^{\prime}(z)$ is starlike with respect to 0 . 
Lemma 2.1 suggests

$$
1+\beta z p^{\prime}(z) \prec 1+\beta z q^{\prime}(z) \Rightarrow p(z) \prec q(z),
$$

so to prove our result, it is suffice to show

$$
s(z)=\frac{1+D z}{1+E z} \prec 1+\beta z q^{\prime}(z)=1+\frac{\beta z}{2 \sqrt{1+z}}=h(z) .
$$

Since $s^{-1}(w)=\frac{w-1}{D-E w}$, then

$$
s^{-1}[h(z)]=\frac{\beta z}{2 \sqrt{1+z}(D-E)-\beta E z} .
$$

For $z=e^{i \theta}, \theta \in[-\pi, \pi]$,

$$
\begin{aligned}
\left|s^{-1}[h(z)]\right| & =\left|s^{-1}\left[h\left(e^{i \theta}\right)\right]\right| \\
& =\frac{\beta}{\left|2 \sqrt{1+e^{i \theta}}(D-E)-\beta E e^{i \theta}\right|} \\
& \geq \frac{\beta}{2\left|\sqrt{1+e^{i \theta}}\right||(D-E)|+\beta|E|} \\
& =\frac{\beta}{2 \sqrt{2 \mid \cos \frac{\theta}{2}}||(D-E)|+\beta| E \mid}
\end{aligned}
$$

It can be shown that the above expression is minimum when $\theta=0$.

Thus

$$
\left|s^{-1}[h(z)]\right| \geq \frac{\beta}{2 \sqrt{2}|(D-E)|+\beta|E|} \geq 1
$$

for $\beta \geq \frac{2 \sqrt{2}|(D-E)|}{(1-|E|)}$. Therefore $\mathbf{D} \subset s^{-1}[h(\mathbf{D})]$ or $s(\mathbf{D}) \subset h(\mathbf{D})$ implies $s(z) \prec h(z)$. Hence, the result is proven.

Corollary 2.1. Let $\beta \geq \beta_{0}, \beta_{0}=\frac{2 \sqrt{2}|D-E|}{(1-|E|)}$ where $-1<E<1,|D| \leq 1$, and $f \in A$.

i) If $f$ satisfies the following

$$
1+\beta \frac{z f^{\prime}(z)}{f(z)}\left(\frac{z f^{\prime \prime}(z)}{f^{\prime}(z)}-\frac{z f^{\prime}(z)}{f(z)}+1\right) \prec \frac{1+D z}{1+E z}
$$


then $f \in S L^{\star}$.

ii) If $1+\beta z f^{\prime \prime}(z) \prec \frac{1+D z}{1+E z} \quad$ then $\quad f^{\prime}(z) \prec \sqrt{1+z}$.

Proof. Define $p(z)=\frac{z f^{\prime}(z)}{f(z)}$ and using Theorem 2.1, the first part of Corollary 2.1 is proved. The second part of our results in Corollary 2.1 can be derived by letting $p(z)=f^{\prime}(z)$.

Theorem 2.2. Let $p$ be an analytic function in $\boldsymbol{D}$ and $p(0)=1$. Let $\beta \geq \beta_{0}, \beta_{0}=$ $\frac{4|D-E|}{(1-|E|)}, \quad-1<E<1$ and $|D| \leq 1$.

$$
1+\beta \frac{z p^{\prime}(z)}{p(z)} \prec \frac{1+D z}{1+E z} \Rightarrow p(z) \prec \sqrt{1+z}
$$

Proof. Let $q(z)=\sqrt{1+z}, q(0)=1$. Elementary calculation will show that $\frac{\beta z q^{\prime}(z)}{q(z)}=\frac{\beta z}{2(1+z)}$ is starlike. Thus, Lemma 2.1 can be applied as

$$
1+\beta \frac{z p^{\prime}(z)}{p(z)} \prec 1+\beta \frac{z q^{\prime}(z)}{q(z)} \Rightarrow p(z) \prec q(z) .
$$

Next, we prove the subordination

$$
\begin{gathered}
s(z)=\frac{1+D z}{1+E z} \prec 1+\beta \frac{z q^{\prime}(z)}{q(z)}=1+\frac{\beta z}{2(1+z)}=h(z) . \\
s^{-1}[h(z)]=\frac{\beta z}{2(1+z)(D-E)-\beta E z} .
\end{gathered}
$$

For $z=e^{i \theta}, \theta \in[-\pi, \pi]$,

$$
\begin{aligned}
\left|s^{-1}[h(z)]\right| & =\left|s^{-1}\left[h\left(e^{i \theta}\right)\right]\right| \\
& =\frac{\beta}{\left|2\left(1+e^{i \theta}\right)(D-E)-\beta E e^{i \theta}\right|} \\
& \geq \frac{\beta}{\left|2\left(1+e^{i \theta}\right)\right||(D-E)|+\beta|E|} \\
& =\frac{\beta}{4\left|\cos \frac{\theta}{2}\right||(D-E)|+\beta|E|}
\end{aligned}
$$

A straight forward computation verifies that the above expression is minimum when $\theta=0$.

Then

$$
\left|s^{-1}[h(z)]\right| \geq \frac{\beta}{4|(D-E)|+\beta|E|} \geq 1
$$

for $\beta \geq \frac{4|(D-E)|}{(1-|E|)}$. Hence $s(\mathbf{D}) \subset h(\mathbf{D})$ implies $s(z) \prec h(z)$. 
Corollary 2.2. Let $\beta \geq \beta_{0}, \beta_{0}=\frac{4|D-E|}{(1-|E|)}, \quad-1<E<1$ and $|D| \leq 1$,

i)

$$
1+\beta\left[1+\frac{z f^{\prime \prime}(z)}{f^{\prime}(z)}-\frac{z f^{\prime}(z)}{f(z)}\right] \prec \frac{1+D z}{1+E z} \Rightarrow f \in S L^{\star}
$$

ii)

$$
1+\beta\left[\frac{(z f(z))^{\prime \prime}}{f^{\prime}(z)}-\frac{2 z f^{\prime}(z)}{f(z)}\right] \prec \frac{1+D z}{1+E z} \Rightarrow \frac{z^{2} f^{\prime}(z)}{f^{2}(z)} \prec \sqrt{1+z} .
$$

Proof. Letting $p(z)=\frac{z f^{\prime}(z)}{f(z)}$ in (i) and $p(z)=\frac{z^{2} f^{\prime}(z)}{f^{2}(z)}$ in (ii) and applying Theorem 2.2 proves the results.

Theorem 2.3. Let $\beta \geq \beta_{0}, \beta_{0}=\frac{4 \sqrt{2}|D-E|}{(1-|E|)}, \quad-1<E<1$ and $|D| \leq 1$.

$$
1+\beta \frac{z p^{\prime}(z)}{p^{2}(z)} \prec \frac{1+D z}{1+E z} \Rightarrow p(z) \prec \sqrt{1+z} .
$$

Proof. Let $q(z)=\sqrt{1+z}$, which implies $\frac{z q^{\prime}(z)}{q^{2}(z)}$ is starlike.

Using Lemma 2.1,

$$
1+\beta \frac{z p^{\prime}(z)}{p^{2}(z)} \prec 1+\beta \frac{z q^{\prime}(z)}{q^{2}(z)} \Rightarrow p(z) \prec q(z) .
$$

Next, let $h(z)=1+\beta \frac{z q^{\prime}(z)}{q^{2}(z)}=1+\frac{\beta z}{2(1+z)^{\frac{3}{2}}}$

$$
s^{-1}[h(z)]=\frac{\beta z}{2(1+z)^{\frac{3}{2}}(D-E)-\beta E z} .
$$

For $z=e^{i \theta}, \theta \in[-\pi, \pi]$,

$$
\begin{aligned}
\left|s^{-1}[h(z)]\right| & =\left|s^{-1}\left[h\left(e^{i \theta}\right)\right]\right| \\
& =\frac{\beta}{\left|2\left(1+e^{i \theta}\right)^{\frac{3}{2}}(D-E)-\beta E e^{i \theta}\right|} \\
& \geq \frac{\beta}{\left|2\left(1+e^{i \theta}\right)^{\frac{3}{2}}\right||(D-E)|+\beta|E|} \\
& =\frac{\beta}{2\left|\left(2 \cos \frac{\theta}{2}\right)^{\frac{3}{2}}\right||(D-E)|+\beta|E|}
\end{aligned}
$$

As in previous case, the above expression is minimum when $\theta=0$. Then

$$
\left|s^{-1}[h(z)]\right| \geq \frac{\beta}{4 \sqrt{2}|(D-E)|+\beta|E|} \geq 1
$$


for $\beta \geq \frac{4 \sqrt{2}|(D-E)|}{(1-|E|)}$. Hence $\mathbf{D} \subset s^{-1}[h(\mathbf{D})]$ implies $s(z) \prec h(z)$.

Corollary 2.3. Let $\beta \geq \beta_{0}, \beta_{0}=\frac{4 \sqrt{2}|D-E|}{(1-|E|)},-1<E<1,|D| \leq 1$ and $f \in A$,

$$
1-\beta+\beta\left[\frac{1+\frac{z f^{\prime \prime}(z)}{f^{\prime}(z)}}{\frac{z f^{\prime}(z)}{f(z)}}\right] \prec \frac{1+D z}{1+E z} \Rightarrow f \in S L^{\star} .
$$

Proof. The result is obtained by taking $p(z)=\frac{z f^{\prime}(z)}{f(z)}$ in Theorem 2.3.

Theorem 2.4. Let $p$ be an analytic function in $\boldsymbol{D}$ and $p(0)=1$.

Let $\beta \geq \beta_{0}, 0<\alpha \leq 1, \beta_{0}=\frac{|1+A||1+B||D-E|}{\alpha|A-B|(1-|E|)}, \quad-1<E<1,|D| \leq 1$ and $-1 \leq B<A \leq 1$.

$$
1+\beta \frac{z p^{\prime}(z)}{p(z)} \prec \frac{1+D z}{1+E z} \Rightarrow p(z) \prec\left(\frac{1+A z}{1+B z}\right)^{\alpha}
$$

Proof. Let $q(z)=\left(\frac{1+A z}{1+B z}\right)^{\alpha}$, Then

$$
\frac{\beta z q^{\prime}(z)}{q(z)}=\frac{\beta \alpha z(A-B)}{(1+A z)(1+B z)}=Q(z)
$$

It can easily be verified that $Q(z)$ is starlike. Lemma 2.1 , we prove the subordination

$$
s(z)=\frac{1+D z}{1+E z} \prec 1+\beta \frac{z q^{\prime}(z)}{q(z)}=1+\frac{\beta \alpha z(A-B)}{(1+A z)(1+B z)}=h(z)
$$

Since $s^{-1}(w)=\frac{w-1}{D-E w}$ then

$$
\begin{aligned}
\left|s^{-1}[h(z)]\right| & =\left|\frac{\beta \alpha z(A-B)}{[(1+A z)(1+B z)(D-E)]-\beta \alpha z E(A-B)}\right| \\
& \geq \frac{|\beta \alpha z(A-B)|}{|[(1+A z)(1+B z)(D-E)]|+|\beta \alpha z E(A-B)|} .
\end{aligned}
$$

For $z=e^{i \theta}, \theta \in[-\pi, \pi]$,

$$
\left|s^{-1}\left[h\left(e^{i \theta}\right)\right]\right| \geq \frac{\beta \alpha|(A-B)|}{\left|\left[\left(1+A e^{i \theta}\right)\left(1+B e^{i \theta}\right)(D-E)\right]\right|+\beta \alpha|E(A-B)|}
$$

with minimum value being attained at $\theta=0$.

Hence

$$
\left|s^{-1}\left[h\left(e^{i \theta}\right)\right]\right| \geq \frac{\beta \alpha|(A-B)|}{|[(1+A)(1+B)(D-E)]|+\beta \alpha|E(A-B)|} \geq 1
$$


for $\beta \geq \frac{|[(1+A)(1+B)(D-E)]|}{\alpha|A-B|(1-|E|)}$ implies $s(z) \prec h(z)$ and the result is obtained.

Remark. Theorem 2.4 is reduced to Theorem 2.2 when $\alpha=\frac{1}{2}, A=1$ and $B=0$.

Finally, we state the next obvious result.

Corollary 2.4. Let $\beta_{0}=\frac{|1+A||1+B||D-E|}{\alpha|A-B|(1-|E|)}, \quad-1<E<1,|D| \leq 1$ and $-1 \leq B<$ $A \leq 1$.

$$
1+\beta\left[1+\frac{z f^{\prime \prime}(z)}{f^{\prime}(z)}-\frac{z f^{\prime}(z)}{f(z)}\right] \prec \frac{1+D z}{1+E z} \Rightarrow \frac{z f^{\prime}(z)}{f(z)} \prec\left(\frac{1+A z}{1+B z}\right)^{\alpha} .
$$

Acknowledgements. This research was supported by IPPP/UPGP/geran(RU/PP P)/PS207/2009A University Malaya grants 2009. The authors are highly grateful to the referees and Prof. Dr. V. Ravichandran for their valuable comments and suggestions.

\section{References}

[1] W. Janowski, Some extremal problems for certain families of analytic functions I, Ann. Polon. Math., 28(1973), 297-326.

[2] S. S. Miller and P. T. Mocanu, Differential subordination, theory and application, Marcel Dekker, Inc., New York, Basel, 2000.

[3] M. Nunokawa, M. Obradović and S. Owa, One criterion for univalency, Proc. Amer. Math. Soc., 106(4)(1989), 1035-1037.

[4] R. M. Ali, N. E. Chu, V. Ravichandran and S. Sivaprasad Kumar, First order differential subordination for functions associated with the lemniscate of Bernoulli, Taiwanese J. Math, 16(3)(2012), 1017-1026.

[5] R. M. Ali, V. Ravichandran and N. Seenivasagan, Sufficient conditions for Janowski starlikeness, International J. Math. and Mathematical Sciences, Article ID 62927(2007), 7 pages.

[6] J. Sokół and J. Stankiewicz, Radius of convexity of some subclasses of strongly starlike functions, Folia Scient. Univ. Tech. Resoviensis, Mat.,19(1996), 101-105.

[7] J. Sokól, Radius problems in the class $S L^{\star}$, Applied Mathematics and Computation, $\mathbf{2 1 4}(2009)$, 569-573.

[8] J. Sokól, Coefficient estimates in a class of strongly starlike functions, Kyungpook Math. J., 49(2009), 349-353.

[9] J. Sokól, On application of certain sufficient condition for starlikeness, J. Math. and Appl., 30(2008), 131-135.

[10] J. Sokół, On sufficient condition to be in a certain subclass of starlike functions defined by subordination, Applied Mathematics and Computation, 190(2007), 237-241. 